\title{
Article \\ A Two-Dimensional Phase-Field Investigation on Unidirectionally Solidified Tip-Splitting Microstructures
}

\author{
V. Pavan Laxmipathy ${ }^{1, *}$, Fei Wang ${ }^{1}$, Michael Selzer ${ }^{1,2} \oplus$ and Britta Nestler ${ }^{1,2}$ \\ 1 Institute of Applied Materials, Karlsruhe Institute of Technology (KIT), Strasse am Forum 7, \\ 76131 Karlsruhe, Germany; fei.wang@kit.edu (F.W.); michael.selzer@kit.edu (M.S.); \\ britta.nestler@kit.edu (B.N.) \\ 2 Institute of Digital Materials Science, Karlsruhe University of Applied Sciences, Moltkestr. 30, \\ 76133 Karlsruhe, Germany \\ * Correspondence: pavan.veluvali2@kit.edu
}

Citation: Laxmipathy, V.P.; Wang, F.; Selzer, M.; Nestler, B. A Two-Dimensional Phase-Field Investigation on Unidirectionally Solidified Tip-Splitting

Microstructures. Metals 2022, 12, 376. https: / /doi.org/10.3390/ met12030376

Academic Editor: Mohsen Eshraghi

Received: 18 January 2022

Accepted: 20 February 2022

Published: 22 February 2022

Publisher's Note: MDPI stays neutral with regard to jurisdictional claims in published maps and institutional affiliations.

Copyright: (C) 2022 by the authors. Licensee MDPI, Basel, Switzerland. This article is an open access article distributed under the terms and conditions of the Creative Commons Attribution (CC BY) license (https:// creativecommons.org/licenses/by/ $4.0 /)$

\begin{abstract}
The onset of morphological instabilities along a solidifying interface has a tendency to influence the microstructural characteristics of cast alloys. In the present study, the initiation as well as the mechanism of microstructural pattern formation is investigated by a quantitative phasefield approach. For energetically isotropic interfaces, we show that the presence of grain boundary grooves promotes the initiation of morphological instabilities, and with progressive solidification, they subsequently amplify into tip-splitting microstructures. We also demonstrate that the grain boundary groove shape influences the amplification of the ridge-shaped instability near the pit region. The structural transition of tip splitting to dendritic microstructures is showcased through the effect of interfacial anisotropy. In addition, the prediction of the tip-splitting position is discussed through an analytical criterion, wherein the sign of the surface Laplacian of interfacial curvature dictates the formation of crest and trough positions in a tip-splitting pattern. In complete agreement with the sharp-interface theory, our phase-field simulations validate the analytically obtained tip-splitting position and suggest that the two tips evolve symmetrically on either side of the hindered concave region. Furthermore, the role of lattice anisotropy on the tip-splitting phenomenon is also discussed in detail.
\end{abstract}

Keywords: pattern formation; tip splitting; phase-field model; grain boundary groove; microstructure formation

\section{Introduction}

Over the last few decades, the selection of microstructural patterns has been a longstanding fundamental interest in scientific and technological advancements. In the presence of high supercoolings [1], pressure gradients [2-4], and low magnetic fields [5], complex microstructural patterns systematically evolve in simulated environments. In unidirectional solidification, the crystal-melt interface generally consists of imperfections in the form of grain boundaries, impurities, and distortions. When morphological instabilities are triggered along a planar front by thermal or constitutional undercooling, these crystal imperfections act as preferred locations for the initiation of interfacial instabilities. Especially, in the presence of grain boundaries, the solid-liquid interfaces become unstable by passing through a different, slightly more complicated, sequence of morphological changes [6]. Schaefer and Glicksman [7] performed seminal experiments and recorded the temporal evolution of morphological instabilities in a polycrystalline sample. Originated near the grain boundary grooves, it was concluded that the protuberances amplified into liquid melt in the form of dendritic branches.

After successful experiments, the stability of a dendritic pattern was first quantitatively evaluated by Mullins and Sekerka [8,9]. It was concluded that an advancing interface splits if the tip radius $\rho_{i}$ is larger than the critical wavelength $\lambda_{i}$. For example, the tip radius 
becomes unstable at the smallest wavelength and results in the formation of symmetrically broken fingers. Later, based on this theory, several investigators $[7,10,11]$ carried out experiments on succinonitrile-based transparent alloys to demonstrate the lateral spreading of the instabilities. For example, Noël et al. [10] performed in situ experiments to study the emergence of non-planar patterns, and they analyzed the cellular interfaces in a transparent binary alloy. It was confirmed that the initiation and the formation of a grain boundary groove lead to the propagation of ridges and depressions along a planar front. In addition, as the solidification velocity was increased beyond the critical velocity of a planar interface, the initial instability occurred at the two slightly convex ridges, which later coarsened and dominated the growth competition among cellular microstructures. Furthermore, at high growth rates, the progressive development of low-amplitude ripples via the formation of ramified patterns was also noticed. Several directional solidification studies [12-14] have shown the emergence of complex tip-splitting microstructures in the absence of interfacial anisotropy. A split tip defines a disordered pattern, whereas the presence of interfacial anisotropy energy brings in a steady and a directionally dependent tip with side branches. Over the last few decades, the structural transition of a solidifying interface into an unidirectionally solidified microstructure has been of considerable interest in cast binary alloys. Therefore, it is important to characterize and control such shapes resulting from solidification.

Apart from the aforementioned literature, various experiments $[13,15,16]$ and theoretical findings [17] have attempted to justify and elucidate the underlying mechanism of branching. While Martin et al. [17] presented the side branching in a dendrite through the disturbances in the mean curvature, Nittman et al. [13] illustrated the tip-splitting phenomenon as a relation with the imposed numerical noise in the bulk. Similarly, Devachelle et al. [18] provided an optimization principle in order to describe the dynamics of a diffusion-limited finger. Moreover, another classical way to study the pattern genesis is through the Hele-Shaw cell [4]. Confined in-between two parallelly separated plates, this method describes the generation of Saffman-Taylor cells, as a result of an unstable interface between the two viscous fluids. Likewise, Suekane et al. [19] studied the three-dimensional characteristics of viscous fingers, where the flow instabilities events were described as a combination of frequent tip splitting, shielding, and coalescence.

More recently, Glicksman [16] proposed a deterministic side-branching mechanism for anisotropic dendrites. According to this theory, the rotation points near the dendritic tip developed a wrinkle, which later grew into a side branch. The local analysis was proved to be successful in kinematically determining the rotation points for branching, wherein the crystal experienced an accelerated growth depending upon the sign of the surface Laplacian of the interfacial curvature $\nabla_{s}^{2} \kappa$. Later, several phase-field simulations [20-22] subsequently confirmed the role of capillary-mediated fluxes in an anisotropic dendrite. In particular, Mullis [20] re-evaluated the findings in Ref. [16] and reported that the above criterion is a useful tool for understanding the branching mechanism. However, in light of the above-mentioned literature, the determination of tip-splitting positions for an energetically isotropic microstructure has been less understood. For example, although previous studies $[23,24]$ have addressed the tip-splitting phenomenon, a quantitative study, whereby the dynamic comparison of the sharp-interface criterion, as well as the role of numerical effects, such as grid anisotropy and numerical noise, is still elusive. Additionally, it needs to be established if the theoretical criterion previously deduced to explain the behavior of secondary branches can be extended to accurately predict the tip-splitting positions. In this regard, the present work intends to fill the gap in our understanding, and it reports a two-dimensional phase-field investigation to elucidate the formation and the behavior of unidirectionally solidified tip-splitting microstructures.

The rest of the manuscript is organized as follows: in the following section, we briefly describe our phase-field model through the evolution equations for the phase and the concentration fields. In the Results and Discussion section, we first simulate the two-dimensional tip-splitting microstructures from a bicrystal specimen. Initiated at the 
grain boundary grooves, we illustrate that the morphological instabilities amplify into tip-splitting microstructures due to the presence of isotropic interfacial energy. Next, the role of groove shape as well as the strength of interfacial anisotropy is discussed in detail. Furthermore, the underlying mechanism of tip instability is discussed in detail, wherein, based on the sharp-interface analytical criterion, the splitting region is showcased. Later, we perform phase-field simulations to demonstrate and validate the analytically derived branching region. In addition, a comparative study between simulations and a sharpinterface theory reveals the effect of the underlying grid on our numerical results. Finally, we put forward the conclusions from the present work.

\section{Model Description}

Over the last two decades, the phase-field approach is gaining popularity as a method of choice to model complex microstructures in solidification, precipitation, and straininduced transformation studies [25]. In the present work, the phase-field model employed is based on the grand potential formulation by Choudhury and Nestler [26] where the evolution of phases is determined by the minimization of the modified functional $d \Omega / d t \leqslant 0$, given by

$$
\Omega(T, \boldsymbol{\mu}, \boldsymbol{\phi})=\int_{\Omega}\left[\Psi(T, \boldsymbol{\mu}, \boldsymbol{\phi})+\left(\epsilon a(\boldsymbol{\phi}, \nabla \boldsymbol{\phi})+\frac{1}{\epsilon} w(\boldsymbol{\phi})\right)\right] d \Omega,
$$

where $T$ is the temperature, $\boldsymbol{\mu}=\left(\mu_{1}, \ldots, \mu_{i}, \ldots, \mu_{K-1}\right)$ is a vector consisting of $K-1$ chemical potentials of the system at a given temperature, and $\boldsymbol{\phi}=\left(\phi_{1}, \ldots, \phi_{\alpha}, \ldots, \phi_{N}\right)$ describes the phase index vector, where $\phi_{\alpha}$ represents the local volume fraction of the $\alpha$ phase. In addition, $\epsilon$ is a length scale related to the thickness of the diffuse interface. The terms $\epsilon a(\boldsymbol{\phi}, \nabla \boldsymbol{\phi})$ and $\frac{1}{\epsilon} w(\boldsymbol{\phi})$ are the gradient and obstacle-type energy densities, which take the thermodynamics of the interface into account [25]. In the present work, the mole fractions of component $B$ in the solid and liquid are set as $c_{B}^{s, e q}=0.8$ and $c_{B}^{l, e q}=0.2$, respectively. Similar to our previous studies $[27,28]$, we consider a binary alloy model system, and we also would like to reiterate that the temporal evolution of tip-splitting microstructures is not affected by the selected mole fractions. Rather, the evolution of the microstructure depends on the imposed melt supersaturation, expressed as $\Delta=\left(c_{B}^{l, e q}-\right.$ $\left.c_{B}^{l}\right) /\left(c_{B}^{l, e q}-c_{B}^{s, e q}\right)$, where $c_{B}^{l}$ is the initial concentration in liquid phase. Lastly, all the parameters used in our numerical simulations are given in Table 1.

Table 1. Dimensionless parameters for the present study.

\begin{tabular}{lcc}
\hline Description & Parameter & Value \\
\hline Partition coefficient & $k$ & 0.25 \\
Discretized grid space & $\Delta x=\Delta y$ & $1.0 \times 10^{-7} \mathrm{~m}$ \\
Domain size & $N x \times N y$ & $1000 \times 1000$ \\
Interface width & $\epsilon$ & $4.0 \times 10^{-7} \mathrm{~m}$ \\
Melt supersaturation & $\Delta=\left(\frac{c_{B}^{l, \text { eq }}-c_{B}^{l}}{c_{B}^{l, e q}-c_{B}^{s, e q}}\right)$ & 0.50 \\
\hline
\end{tabular}

The evolution equations for the phase-field is given as

$$
\tau \epsilon \frac{\partial \phi_{\alpha}}{\partial t}=\epsilon\left(\nabla \cdot \frac{\partial a(\boldsymbol{\phi}, \nabla \boldsymbol{\phi})}{\partial \nabla \phi_{\alpha}}-\frac{\partial a(\boldsymbol{\phi}, \nabla \boldsymbol{\phi})}{\partial \phi_{\alpha}}\right)-\frac{1}{\epsilon} \frac{\partial w(\boldsymbol{\phi})}{\partial \phi_{\alpha}}-\frac{\partial \Psi(T, \mu, \boldsymbol{\phi})}{\partial \phi_{\alpha}}-\lambda,
$$

where $\lambda$ is a Lagrange multiplier so that the local constraint $\sum_{\alpha=1}^{N} \phi_{\alpha}=1$ is fulfilled. The gradient energy term reads as,

$$
\epsilon a(\boldsymbol{\phi}, \nabla \boldsymbol{\phi})=\epsilon \sum_{\alpha, \beta=1}^{N, N} \gamma_{\alpha \beta}\left[a_{c}\left(\boldsymbol{q}_{\alpha \beta}\right)\right]^{2}\left|\boldsymbol{q}_{\alpha \beta}\right|^{2}
$$


where $q_{\alpha \beta}$ models the surface energy of the evolving phase boundary and $\gamma_{\alpha \beta}$ is the interfacial energy per unit area of the $\alpha / \beta$ interface. Here, to simulate a tip-splitting microstructure, an isotropic interfacial energy is imposed using $a_{c}\left(\boldsymbol{q}_{\alpha \beta}\right)=1$. Interfaces with finite interfacial anisotropy and having an underlying four-fold cubic symmetry are modeled by the expression

$$
a_{c}\left(\boldsymbol{q}_{\alpha \beta}\right)=1-\delta_{\alpha \beta}\left(3 \mp 4 \frac{\left|\boldsymbol{q}_{\alpha \beta}\right|_{4}^{4}}{\left|\boldsymbol{q}_{\alpha \beta}\right|^{4}}\right),
$$

where $\left|\boldsymbol{q}_{\alpha \beta}\right|_{4}^{4}=\sum_{i=1}^{d}\left(q_{i}^{4}\right)$ and $\left|\boldsymbol{q}_{\alpha \beta}\right|^{4}=\left[\sum_{i=1}^{d}\left(q_{i}^{2}\right)\right]^{2}$. The strength of the anisotropy of the $\alpha-\beta$ phase or grain boundary is given by the parameter $\delta_{\alpha \beta}$. In general, phase transformation in alloy solidification is driven by both heat and mass transport. However, since these processes occur at significantly different time scales, for the present study, the conduction of heat in the system is treated as isothermal, wherein the domain temperature $T$ in Equation (2) is assumed to be constant throughout the system and thereby plays no role in the temporal evolution of the microstructures. The term $w(\phi)$ represents the multiobstacle potential. Now, the evolution equation for the chemical potentials can be expressed as

$$
\frac{\partial \mu}{\partial t}=\left[\sum_{\alpha=1}^{N} h\left(\phi_{\alpha}\right) \frac{\partial c(\boldsymbol{\mu})}{\partial \mu}\right]^{-1} \times\left\{\nabla \cdot\left(M(\boldsymbol{\phi}) \nabla \mu-J_{a t}-\boldsymbol{q}\right)-c^{\alpha}(\boldsymbol{\mu}) h^{\prime}\left(\phi_{\alpha}\right) \frac{\partial \phi}{\partial t}\right\} .
$$

In the above equation, the mass flux on the right-hand side has contributions from diffusion due to the gradient in the chemical potential and the interface mobility $M_{i j}(\phi)$. Additionally, with an imposed magnitude of 0.06 , the term $q$ represents the conserved noise in the bulk liquid phase, and the anti-trapping current $J_{a t}$ compensates the solute-trapping effect due to an enlarged interface in our phase-field model. This effect can be negated through the anti-trapping current; see Choudhury and Nestler [26] for more details. All terms in Equations (2) and (5) are explicitly defined by Choudhury and Nestler [25,26]. Lastly, while Equations (2) and (5) are numerically solved in space via a finite difference discretization, the time derivative follows an explicit Euler scheme. Finally, the current numerical algorithm is parallelized via message passing interface (MPI) to distribute the computing task on multicore high-performance architectures.

\section{Results and Discussion}

\subsection{Tip-Splitting Microstructures}

Initiated at the grain boundary, we first investigate the temporal evolution of a morphological pattern, wherein the planar solidification front transforms into a ramified tipsplitting microstructure in a supersaturated melt under isothermal conditions; see Figure 1. Here, we perform the numerical simulations in a two-dimensional domain of $1000 \times 1000$ numerical cells, with the space discretization $\Delta x=1.0 \times 10^{-7} \mathrm{~m}$. In addition, the interface width is given as $\epsilon=4 \times \Delta x$, such that the equilibrium diffuse-interface profile encompasses eight grid points in the numerical domain. Furthermore, on the left and right side of the domain walls, the periodic boundary condition is imposed for the phase and concentration fields. At the top boundary, the Neumann boundary condition is applied for both the fields.

In a supersaturated melt of composition $\Delta=0.50$, and during the initial stages, the interface remains planar and advances slowly toward the liquid region. As shown in Figure 1, with the accumulation of solutal fields ahead of the solid-liquid interface at the groove pit, the planar interface loses its stability and undergoes the well-known MullinsSekerka instability [8], which states that the stability of a solid-liquid interface is governed by the critical wavelength and the extent of constitutional supercooling in the bulk. When compared with the grain centers, the instabilities at the grain boundary amplify at a faster rate into primary ridges. We also notice that parts of the grain centers not immediately 
adjacent to the grain boundary remain featureless and undistorted. Afterwards, while the shape of the instability across the domain depends on whether it decays or grows, the initial morphological instability from a liquid groove always coarsens. As a ridge is developed near a grain boundary, the solute is laterally rejected outwards, leading to the formation of secondary depressions. Nevertheless, these primary ridges evolve into a periodic array of hills and later transform into a ramified dendritic network, depending upon the anisotropy in the solid-liquid interfacial energy. Analogous to the present observation, the correlation between grain boundaries and the formation of cellular arrays was reported in the experimental work of Noël et al. [10]. It was suggested that the surface undulations and cell alignment along the grain boundary, typically observed during the initial stages, give way to a flat, regular arrangement of cells. Moreover, in the present scenario and as depicted in Figure 1, the dynamic evolution of the interface instabilities is simulated with isotropic solid-liquid energy such that random tip-splitting structures are observed. The simulated non-planar tip-splitting microstructures are widely known as seaweed structures, which were originally observed in several experimental and phase-field investigations $[1,2,12,13,29]$. While the columnar dendrites are directionally dependent with a steady-state dendritic shape, the ramified microstructures in Figure 1 are considered as solidification patterns without any orientational order. Only from a morphological point of view these tip-splitting microstructures look like the degenerate tips in a directionally solidified dendrite [30].
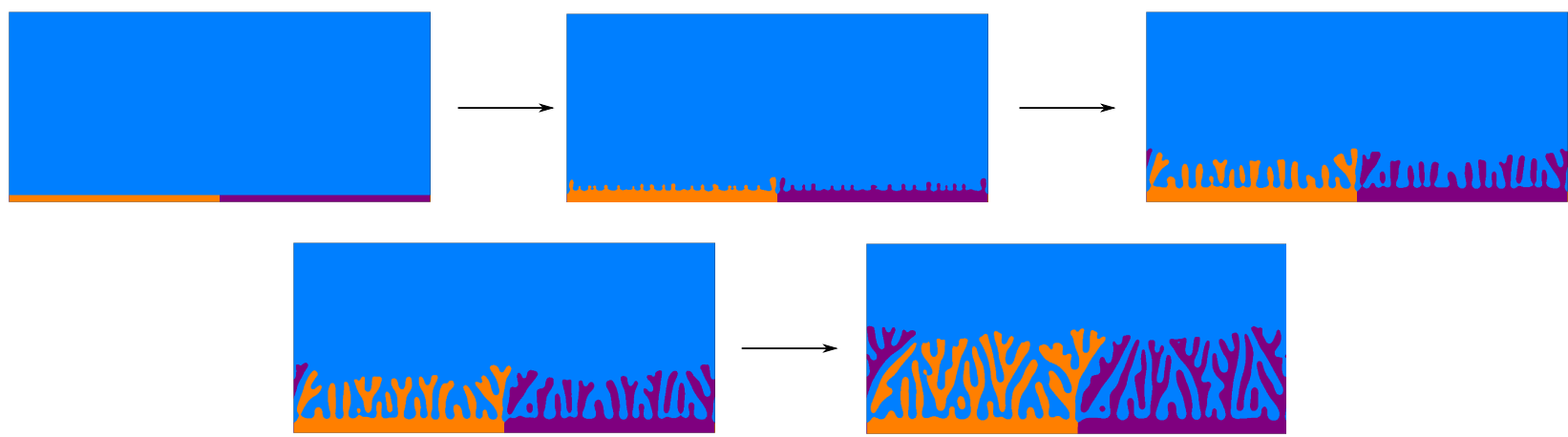

Figure 1. Temporal evolution of a tip-splitting microstructure in a supersaturated melt of composition $\Delta=0.50$. The primary ridges developed at the grain boundary groove amplify into split structures along with the propagation of hillocks across the two solid grains. The combined influence of constitutionally supercooled alloy melt near the solid-liquid interface and isotropic interfacial energy promote the formation of a tip-splitting morphology. Here, the magenta and orange colors refer to the two distinct solid grains, and blue color represents the liquid phase, respectively.

Depicted in Figure 2a-c, it is important to note that the fundamental and repeating characteristic of a seaweed structure is the successive and continuous splitting of the tips. Herein, a local competition between the preferred crystal growth direction and the presence of strong concentration gradients results in the repeated splitting of the leading tip into two parts. In Figure 2a, when growth conditions are imposed for which a planar interface just becomes unstable, a zone of supercooled liquid exists ahead of the planar interface, which causes the interface to become unstable and to form a perturbed shape. As the threshold value of the interface velocity is exceeded, the lateral diffusion of solute reduces the solute concentration at the cell tip. Subsequently, with progressive solidification, this sequential process is repeated, and thereafter, a complex fractal-like dense microstructure is generated. Similar to the diffusion-limited growth of isotropic crystals $[2,12,13]$, the formation of seaweed patterns is inherently related to the low anisotropic properties of the solid-liquid interface. Nevertheless, the absence of anisotropy promotes an uninhibited, omnidirectional growth via irregular branching and splittings of an evolving interface [24]. 

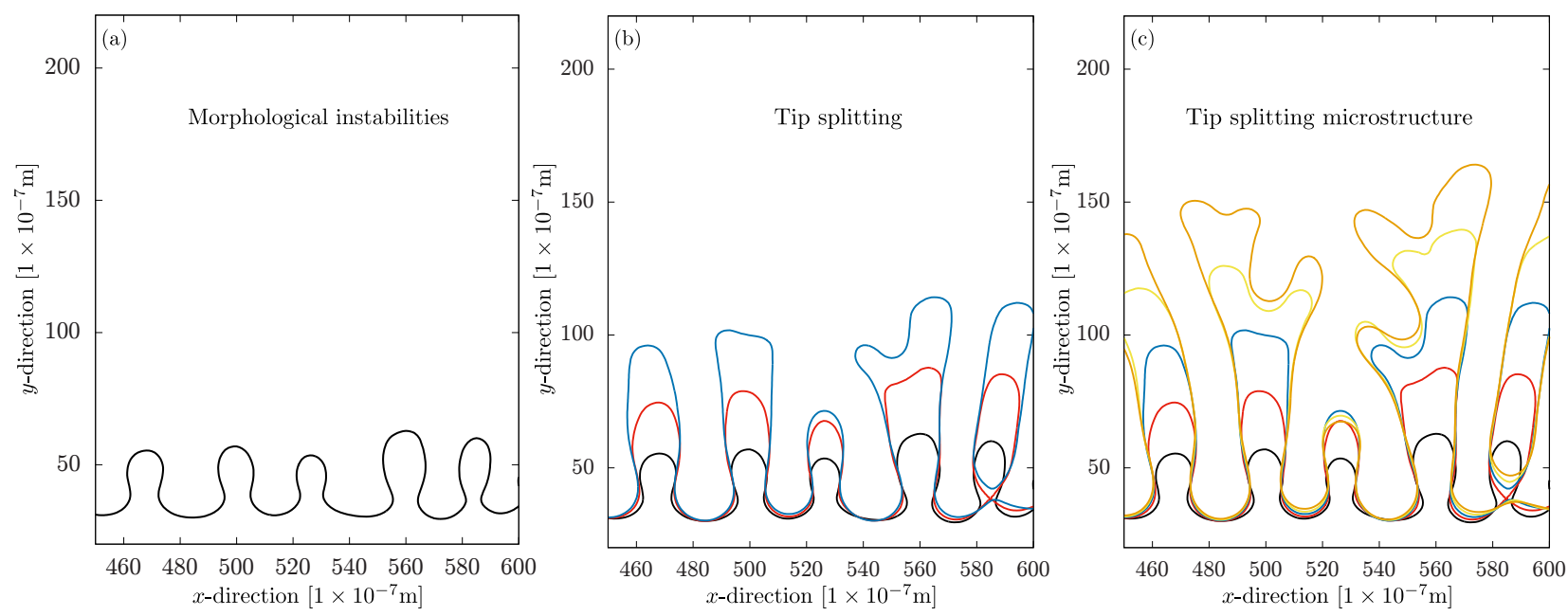

Figure 2. (a-c) Phase-field isolines illustrating the development of a tip-splitting microstructure from a unidirectionally solidified solid-liquid interface. Morphological instabilities evolve with time to undergo successive branching at the tip. Sequential steps to generate a seaweed microstructure: Initial morphological instabilities $\rightarrow$ amplification of tips $\rightarrow$ tip-splitting event. Isolines with various colors represent different simulation timesteps.

\subsection{Effect of Groove Shape}

Grain boundary grooves develop along a solidification front whenever bicrystal or polycrystal grains are in close contact with an alloy melt. Since the grain boundary groove region initiates pattern formation, in this section, we study the role of groove shapes on the morphological development of instabilities during alloy solidification. Figure 3 depicts the temporal inception of a ridge-shaped instability near a groove pit for different $R_{0}$, where $R_{0}$ is the ratio between solid-solid $\gamma_{\mathrm{gb}}$ and solid-liquid interfacial energies $\gamma_{\mathrm{sl}}$. As shown in Figure 3, we observe that an increase in the ratio $R_{0}$ amplifies the growth of the instability adjacent to the grain boundary groove region. As the solidification of the solid-liquid interface commences from an initial configuration, the imposed surface energy modifies the groove shape, which in turn assists in the temporal development of ridges into tipsplitting microstructures. With increasing solid-liquid interfacial energy contribution, i.e., for decreasing $R_{0}$, and as shown via Figure 4 , we also notice that a shallow groove shape promotes the constrained growth of humps such that the instability height decreases as a function of simulation time. This behavior qualitatively agrees with previous experimental and phase-field studies [7,31], where the role of a grain boundary groove on the initiation of morphological instabilities during alloy solidification was discussed in detail. Furthermore, the stability analysis by Coriell et al. [32] also provided a theoretical background. It was reported that the groove pit depth determines the transient amplification of the ridges, and the main role of a grain boundary is to provide an initial perturbation based on the local concentration gradients at the pit region. Therefore, the formation of an instability near the grain boundary groove and the initiation of perturbations across the solid grains is largely controlled and influenced by the chosen surface energies at the trijunction. Nonetheless, for the limiting case $R_{0} \rightarrow 0$, as the grain boundary energy contribution diminishes, we expect the development of an instability to be completely inhibited. 

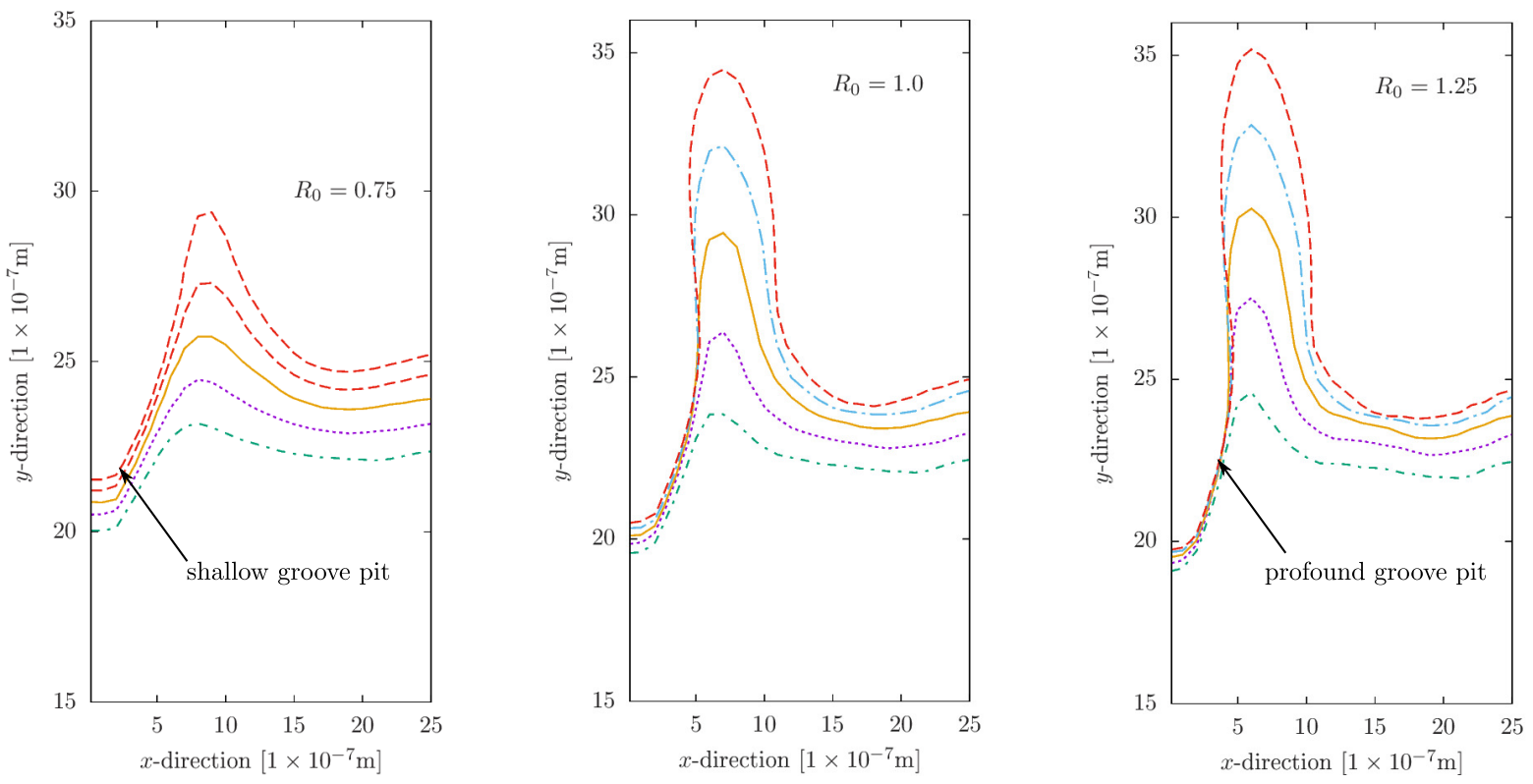

Figure 3. Effect of groove shape on the morphological development of primary ridges near a grain boundary. An increase in the ratio $R_{0}$, where $R_{0}$ is the ratio between solid-solid $\gamma_{\mathrm{gb}}$ to solid-liquid interfacial energies $\gamma_{\mathrm{sl}}$ increases the local amplification of the ridge shaped instability. In contrast, a shallow groove due to an increase in the solid-liquid interfacial energy contribution (decreasing $R_{0}$ ) hinders the growth of the instability near the groove pit region. Various colored isolines represent different simulation timesteps.

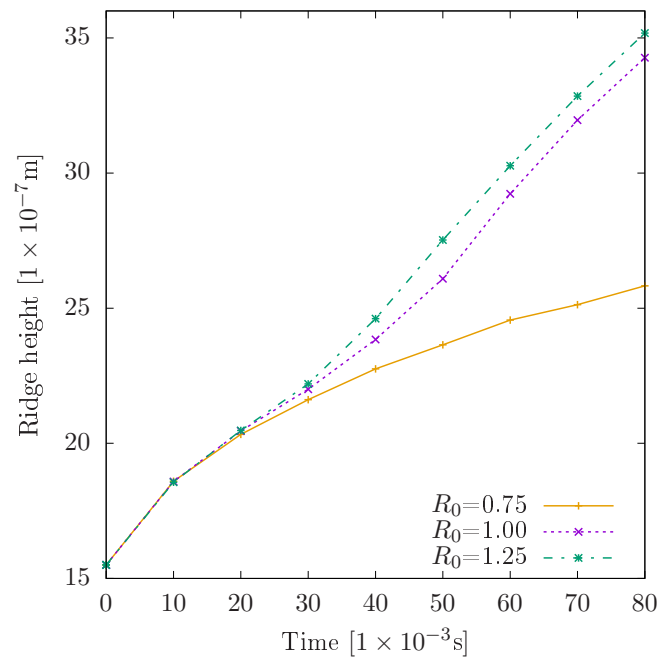

Figure 4. Ridge height as a function of simulation time for different groove shapes (different $\left.R_{0}\right)$. An increase in the ratio $R_{0}$ assists the morphological development of ridge-shaped instability near the grain boundary groove region.

\subsection{Effect of Interfacial Anisotropy}

In this section, we briefly study the role of interfacial anisotropy on pattern formation, and the various morphologies that arise from an unidirectionally solidified interfacial front are depicted in Figure 5. Here, we impose a four-fold interfacial anisotropy in solid-liquid energy formulation such that the strength of anisotropy of an $\alpha / \beta$ interface is given by the parameter $\delta_{\alpha \beta}$; for expression and implementation, see Ref. [25]. As shown in Figure 5, we observe that the imposed strength of anisotropy controls the direction as well as the morphology of the microstructure. While $\delta_{\alpha \beta}=0.0$ generates a disoriented tip-splitting structure, an increase in the anisotropic strength $\delta_{\alpha \beta}$ promotes the formation of steady- 
state columnar dendrites. In general, the local competition of bulk and interfacial forces determines the transition of a planar interface into a ramified pattern. Due to the presence of significant surface energy anisotropy, the absence of frequent tip splitting is noticed for $\delta_{\alpha \beta}>0.01$. As reported in our previous study [28], the presence of a grain boundary promotes an inter-dendritic growth competition among converging dendritic networks, which in turn influences the texture as well as the structural stability of single-crystal alloys in high-temperature applications.

$\delta_{\alpha \beta}=0.0$

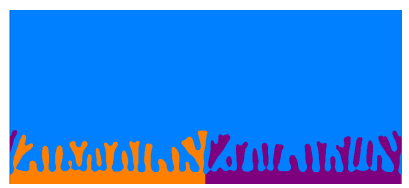

$\delta_{\alpha \beta}=0.005$

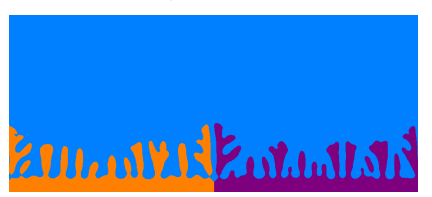

$\delta_{\alpha \beta}=0.02$

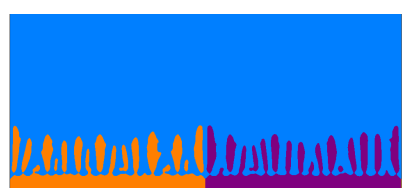

$\delta_{\alpha \beta}=0.03$

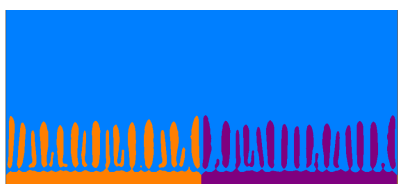

Increasing anisotropic strength

Figure 5. Reconstructed simulation screenshots illustrating the role of interfacial anisotropy on pattern formation. With an increase in the anisotropic strength $\delta_{\alpha \beta}$, the tip-splitting microstructure translates into an array of columnar dendrites.

\subsection{Analytical Criterion for Tip Splitting}

From the aforementioned simulations, it is evident that for a tip-splitting morphology, the stability ranges exist over a wide range of growth conditions, and the solidification patterns evolve into dendrites or seaweeds depending upon the imposed interfacial anisotropy [33]. However, one of the major drawbacks in the literature is that such a study shows no clarity on the tip-splitting position. Recently, Glicksman [16] proposed an analytical criterion for the branching mechanism through the Gibbs-Thomson temperature distribution as an active interfacial energy. According to this theory, the sign of the surface Laplacian of interfacial curvature $\nabla_{S}^{2} \kappa$, where $\kappa$ is the interfacial curvature, predicts whether the interface moves toward the melt or the crystal. It was reported that a positive $\nabla_{S}^{2} \kappa$ represented the growth of the interface, whereas a negative $\nabla_{S}^{2} \kappa$ promoted a hindered growth of the interface [16]. Thereupon, the local analysis was proved to be successful in kinematically determining the rotation points for branching, where the crystal experienced an accelerated growth. The present section builds on this theory and addresses the fundamental tip-splitting behavior in seaweed microstructures. In the forthcoming segments, the expressions for the sharp-interface analytical criterion for a convex profile in two dimensions is derived and subsequently compared with two-dimensional phase-field simulations.

Since the main objective of this section is to demonstrate and predict the tip-splitting phenomenon, similar to the instabilities observed in the Figure 2a, the interface shape is chosen to be convex and symmetric in order to avoid any mathematical complexity. To begin with, the analytical criterion for a two-dimensional surface is determined in the following manner. Let $f(x)$ be the equation of a parabola in a two-dimensional domain, given as

$$
f(x)=-\alpha(x-h)^{2}+p,
$$

where $\alpha, h$, and $p$ are the scaled dimensionless parameters controlling the width and the position of the parabola. In the present study, $h=500$ and $p=250$, and $\alpha$ is taken as 0.02 . Let us begin the calculation of the analytical criterion $\nabla_{S}^{2} \kappa$, starting with the expression of interfacial curvature $\kappa(x)$, for a two-dimensional convex interface,

$$
\kappa(x)=\frac{f^{\prime \prime}(x)}{\left(1+f^{\prime}(x)^{2}\right)^{\frac{3}{2}}} .
$$


Now, the gradient of the interfacial curvature along the arc length $s$ can be written as

$$
\begin{gathered}
\frac{\partial \kappa}{\partial s}=\frac{\partial \kappa}{\partial x} \frac{\partial x}{\partial s} \\
\frac{\partial \kappa}{\partial s}=\frac{1}{\sqrt{1+\left(\frac{\partial y}{\partial x}\right)^{2}}} \frac{\partial \kappa}{\partial x} .
\end{gathered}
$$

Let the above equation be termed as $g(x)$; thus,

$$
g(x)=\frac{1}{\sqrt{1+\left(\frac{\partial y}{\partial x}\right)^{2}}} \frac{\partial \kappa}{\partial x} .
$$

Finally, in order to calculate the surface Laplace, the surface divergence of the gradient along the arc length is taken. Using Equation (10), we obtain

$$
\nabla_{s}^{2} \kappa=\frac{\partial g(x)}{\partial x} \frac{\partial x}{\partial s}=\frac{1}{\sqrt{1+\left(\frac{\partial y}{\partial x}\right)^{2}}} \frac{\partial g(x)}{\partial x} .
$$

The above derivation can be rewritten by substituting $f(x)$ from Equation (6) into Equation (7) as

$$
\nabla_{s}^{2} \mathcal{K}=\frac{\frac{576 \alpha^{5}(x-h)^{2}}{\left(1+4 \alpha^{2}(x-h)^{2}\right)^{4}}-\frac{24 \alpha^{3}}{\left(1+4 \alpha^{2}(x-h)^{2}\right)^{3}}}{\sqrt{1+4 \alpha^{2}(x-h)^{2}}} .
$$

The positions along the $x$-direction for which the analytical criterion is exactly zero are given by

$$
x=\frac{2 h \alpha \pm \frac{\sqrt{5}}{5}}{2 \alpha} .
$$

As shown in Figure 6, Equation (12) represents the analytically predicted tip-splitting position for a convex crystal-melt interface. Herein, we observe that the sharp-interface criterion $\nabla_{s}^{2} \kappa$ for $\alpha=0.02$ has two positive regions at $x<490 \times 10^{-7} \mathrm{~m}$ and $x>510 \times 10^{-7}$ $\mathrm{m}$, whereas the negative region lies in between $490 \times 10^{-7} \mathrm{~m}<x<510 \times 10^{-7} \mathrm{~m}$. During solidification, and as represented with a red dot in Figure 6, the minimum at $\nabla_{s}^{2} \kappa=-0.000191$ starts to evolve toward the solid in a hindered manner. In contrast, we notice that the two maxima at $\nabla_{s}^{2} \kappa=4.6 \times 10^{-5}$ (black dots in Figure 6) shall accelerate toward the opposite direction in order to generate the foremost point. Therefore, the tip-splitting region is predicted where the interface undergoes a hindered growth, and it begins when the analytical criterion passes through zero. The form of the plot in Figure 6 is a direct comparison with the normal flux reported by Glicksman [16], where the solid-liquid interface evolved accordingly to undergo systematic branching. Nonetheless, having derived the analytical positions for a tip-splitting event, in the next section, we systematically compare with our phase-field results. 


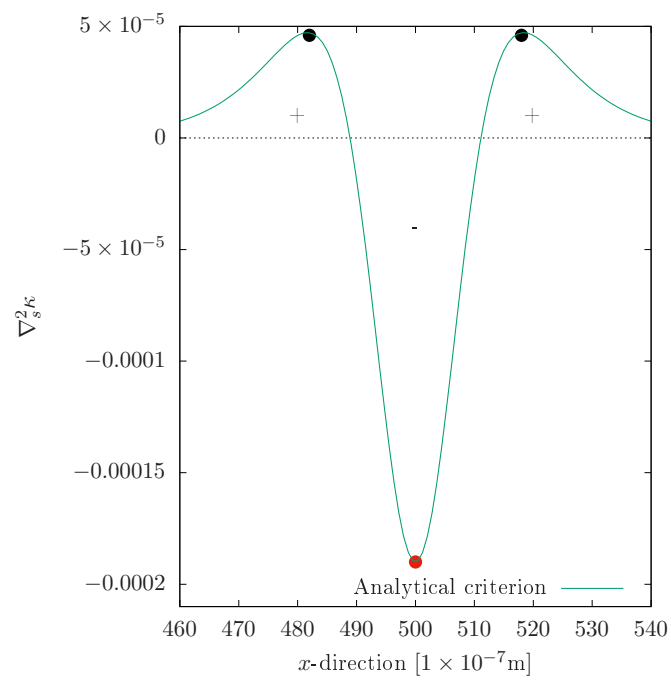

Figure 6. Calculated sharp-interface analytical criterion for $\alpha=0.02$ as a function of the grid position. The positive and negative regions indicate the crest and the trough positions during a tip-splitting event. The forked region lies between the $\nabla_{s}^{2} \kappa<0$ region, whereas the two tips start to evolve at the $\nabla_{s}^{2} \kappa>0$ positions.

\subsection{Comparison between Sharp-Interface Analytical Criterion and Phase-Field Simulation}

In the present section, two-dimensional phase-field simulations are performed to comprehend and subsequently validate the analytically derived tip-splitting position. As shown earlier and in previous studies [29,33], crystal-melt interfaces with weak anisotropies evolve into tip-splitting microstructures in a unidirectionally solidification setup. Similar to previous sections, the domain size as well as the boundary conditions are considered to be the same; i.e., on the left and right side domain walls, the periodic boundary condition is imposed for the phase and concentration fields. At the top boundary, the Neumann boundary condition is applied for both the fields. In order to have a direct comparison with the sharp-interface criterion, the initial condition is a convex crystal-melt interface profile with $\delta_{\alpha \beta}=0.005$ as given by Equation (6), and the temporal evolution of the solid-liquid interface is shown in Figure 7 via the red isolines. At $t=130 \times 10^{-3} \mathrm{~s}$, the red isoline in Figure 7 depicts the formation of a crest and trough, which in turn indicates that the evolving crystal-melt interface has undergone tip splitting. The projection of Figure 6 (dashed blue line) in Figure 7 (solid red line) further reveals that the interface evolves into a crest where the analytical criterion is positive, while the negative $\nabla_{s}^{2} \kappa$ corresponds to a hindered forked region. In Figure 7, it can be seen that this segment now proceeds in the opposite direction toward the crystal, compared with the two hillocks developed on either side, when $\nabla_{s}^{2} \kappa$ passes through zero. Such positions are known as Laplace or rotation points; i.e., the sign of the analytical criterion $\nabla_{s}^{2} \kappa$ reverses [24]. As derived earlier, the interface positions where the analytical criterion is exactly zero are given by Equation (13), and a direct comparison with the phase-field result also shows a convincing agreement with the splitting positions in Figure 7. Further comparisons with different $\alpha$ are included in the supplemental material. The present analysis agrees in spirit with Kesseler, Koplik, and Levine $[34,35]$, where a geometrical approach successfully proved that the interfacial curvature at the tip decreases with time, and finally, a tip-splitting event is initiated once the interfacial curvature at the tip passes through zero. Additionally, a sharp-interface criterion for side branching in anisotropic dendrites was reported with similar remarks [17]. Here, the evolution of a convex instability was tracked, wherein the difference between the time-dependent curvature and the curvature of a steady-state crystal was plotted to present different scenarios for the generation of side branches. 


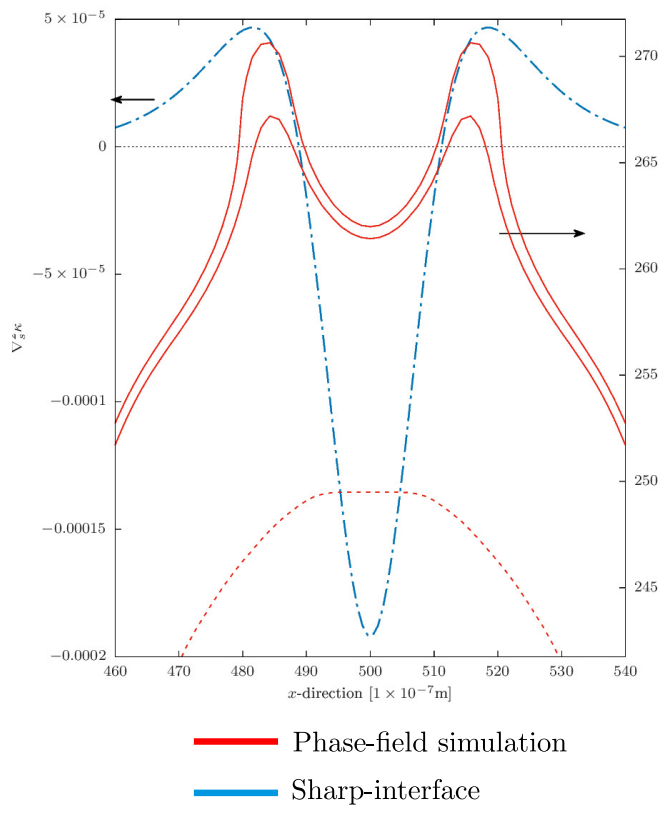

Figure 7. The temporal formation of crests, at $x=490 \times 10^{-7} \mathrm{~m}, x=510 \times 10^{-7} \mathrm{~m}$, and the formation of a trough at $490 \times 10^{-7} \mathrm{~m}<x<510 \times 10^{-7} \mathrm{~m}$ exhibit the tip-splitting phenomenon from a two-dimensional phase-field simulation (red isolines). The initial interface profile in accordance with Equation (6) is also illustrated. A direct comparison with the analytically obtained positions (blue dashed lines) reveal that both tips are generated in the positive regions of Figure 6, whereas the split region is determined by the negative region of the interface. The left and right arrows respectively indicate the $\nabla_{s}^{2} \kappa$ and the grid positions.

In general, the magnitude of fluxes plays an important role in the formation of tipsplitting microstructures. A recent study by Mullis [20] found the magnitude of tangential flux and location of the first perturbation to be consistent at high undercoolings. For a solid-liquid interface profile, where the interfacial curvature varies non-linearly with its arc length, the gradients developed parallel to the interface contribute toward the generation of an inflection point, especially at the tip region. However, as these fluxes are proportional to the interfacial curvature, they recede significantly away from the tip, where the bulk fluxes are dominant. The surface accelerates due to cooling and retards by virtue of heating; a combination of such effects creates the development of an inhibited interface at the negative $\nabla_{s}^{2} \kappa$ region, facilitating the branching process at the predicted position. In other words, the initiation of the cusped region transpires when $\nabla_{s}^{2} \kappa$ turns negative. The concave portion of the interface in Figure 7 also indicates some sort of solutal sink along the U-shaped interface. Finally, the two tips that are triggered by means of strong fluxes multiply into several tip-splitting events during the later stages of the simulation and produce a classic seaweed microstructure.

While we have discussed the initiation and the mechanism of a tip-splitting event, the absence of such instabilities is also observed when a sphere is considered as an initial condition; see Figure 8. For a sphere with an isotropic interfacial energy, the curvature-dependent analytical criterion is defined by the absence of crest and trough regions. Likewise, with such an initial condition, the sphere evolves consistently without any tip-splitting events, and the simulated isotropic spherical seed in Figure 8 is in excellent agreement with the sharp-interface theory. Lastly, while the role of lattice anisotropy is investigated in the upcoming section, the role of discretization spacing is discussed in detail in the supplementary material. 

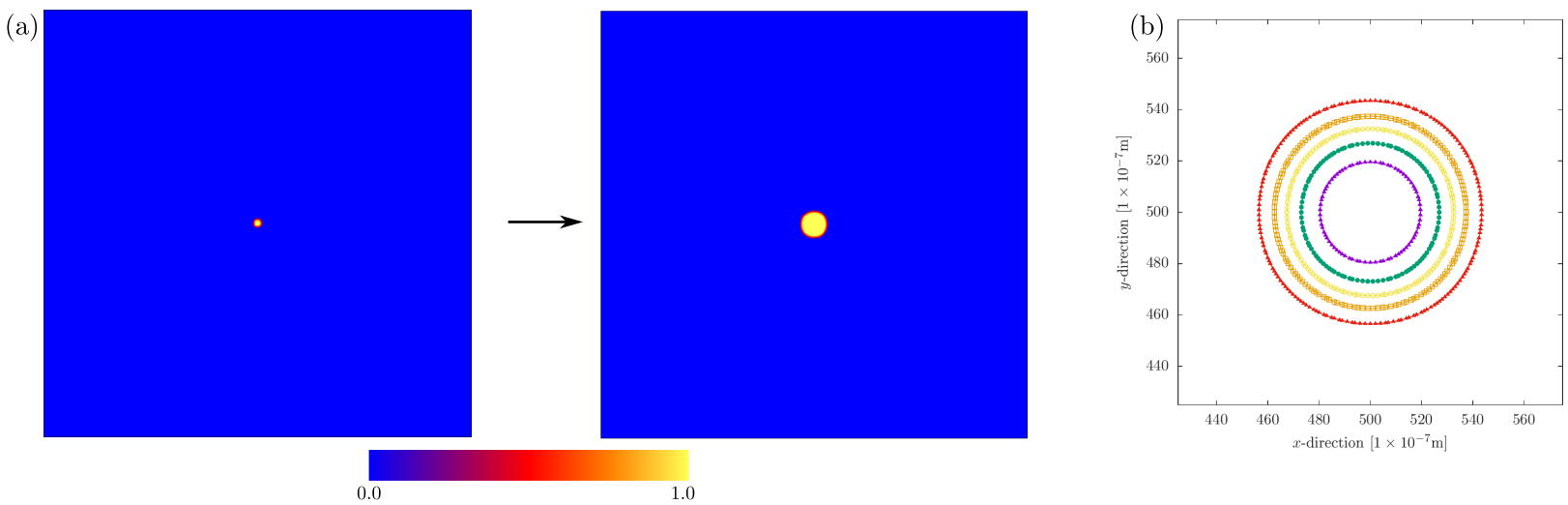

Figure 8. $(\mathbf{a}, \mathbf{b})$ Simulation screenshots and phase-field isolines for an isotropic spherical seed. As the interfacial curvature varies linearly with its arc length, the absence of tip splitting is also captured. This phenomenon is in complete accordance with the sharp-interface analytical prediction, which is defined by the absence of crest and trough regions. The color bar illustrates the phase-field according to the legend embedded below the simulation snapshots. Left to right simulation frames, as well as the phase-field isolines, indicate the evolution of the spherical seed with time.

\subsection{Effect of Lattice Anisotropy}

In the present section, the effect of lattice anisotropy on our numerical results is discussed in detail. In order to accurately analyze the tip-splitting phenomenon and minimize the pinning effect of the underlying lattice on the solid-liquid interface, we systematically compare the equilibrium shapes of a spherical crystal seed from the phasefield simulation with its corresponding shape in the sharp-interface theory [36,37], which is expressed in the Cartesian coordinates as

$$
\begin{gathered}
x=r_{0}\left[f(\theta, \varphi) \sin (\theta) \cos (\varphi)+\frac{\partial f(\theta, \varphi)}{\partial \theta} \cos (\theta) \cos (\varphi)-\frac{\partial f(\theta, \varphi)}{\partial \varphi} \sin (\varphi) / \sin (\theta)\right], \\
y=r_{0}\left[f(\theta, \varphi) \sin (\theta) \sin (\varphi)+\frac{\partial f(\theta, \varphi)}{\partial \theta} \cos (\theta) \sin (\varphi)+\frac{\partial f(\theta, \varphi)}{\partial \varphi} \cos (\varphi) / \sin (\theta)\right], \\
z=r_{0}\left[f(\theta, \varphi) \cos (\theta)-\frac{\partial f(\theta, \varphi)}{\partial \theta} \sin (\theta)\right],
\end{gathered}
$$

and,

$$
f(\theta, \varphi)=1+\frac{4 \delta_{\alpha \beta}^{e}}{1-3 \delta_{\alpha \beta}^{e}}\left[\cos ^{4}(\theta)+\sin ^{4}(\theta)\left(1-2 \sin ^{2}(\varphi) \cos ^{2}(\varphi)\right)\right] .
$$

Here, $(\theta, \varphi)$ are the spherical angles along the normal direction to the solid-liquid interface. Since our study is focused on two-dimensional simulations, we restrict ourselves by comparing the equilibrium shapes along the $x-y$ plane only. Furthermore, in the above equation, $\delta_{\alpha \beta}^{e}$ represents the effective anisotropic strength of the solid-liquid interface, given as $\delta_{\alpha \beta}^{e}=\frac{r_{10} / r_{11}-1}{r_{10} / r_{11}+1}$, where $r_{10}$ and $r_{11}$ are the radial distances from the origin to the solid-liquid interface along the $y$ axis, and along the $x=y$ line, respectively, from the phase-field simulation.

Next, the equilibrium shape obtained from our phase-field study is first initialized with a two-dimensional seed of radius $r_{0}=45 \times 10^{-7} \mathrm{~m}$. In addition, the melt supersaturation $\Delta=0.8$ is selected such that the bulk phases are in equilibrium, and the solid phase would neither shrink nor grow with time. As shown in the earlier section, the tip-splitting microstructures are widely observed for $\delta_{\alpha \beta}<0.01$, and thereby, we have considered two different anisotropic strengths, $\delta_{\alpha \beta}=0$ for the isotropic case, and $\delta_{\alpha \beta}=0.005$ for weakly anisotropic interfaces. Figures 9 and 10 depict the equilibrium shapes from the phase-field simulations, and an excellent agreement with the obtained sharp-interface profiles for 
two different cases is observed. Henceforth, from the the above analysis, we emphasize that the grid or lattice anisotropy has a minimal effect on the crystal-melt shapes, which quantitatively illustrates the accuracy of our phase-field results. Nevertheless, for the sake of further investigating the role of lattice anisotropy, direct comparisons for simulations with strong anisotropic strengths and different crystal radii have been discussed in detail in the supplementary material.

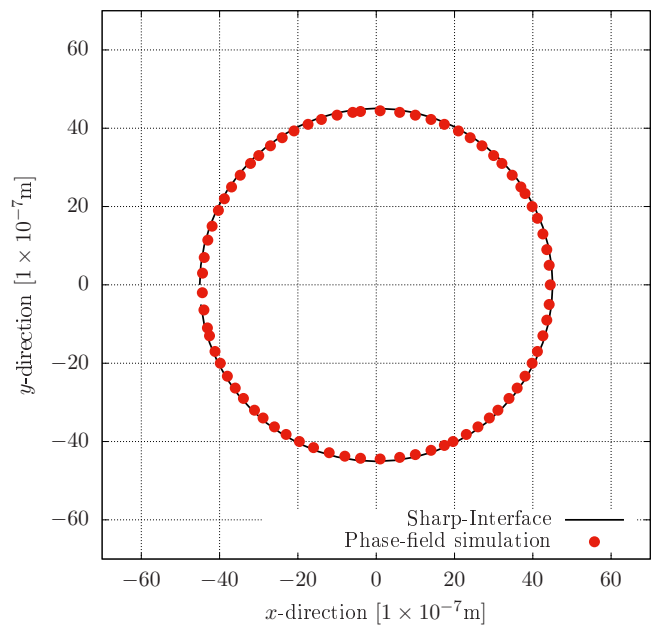

Figure 9. For an energetically isotropic interface $\delta_{\alpha \beta}=0$, the simulated solid phase with radius $r_{0}=45 \times 10^{-7} \mathrm{~m}$ shows a complete agreement with the sharp-interface theory. Here, the obtained effective anisotropy $\delta_{\alpha \beta}^{e}=0.0001$ shows negligible effect from the underlying lattice on our simulated crystal shape.

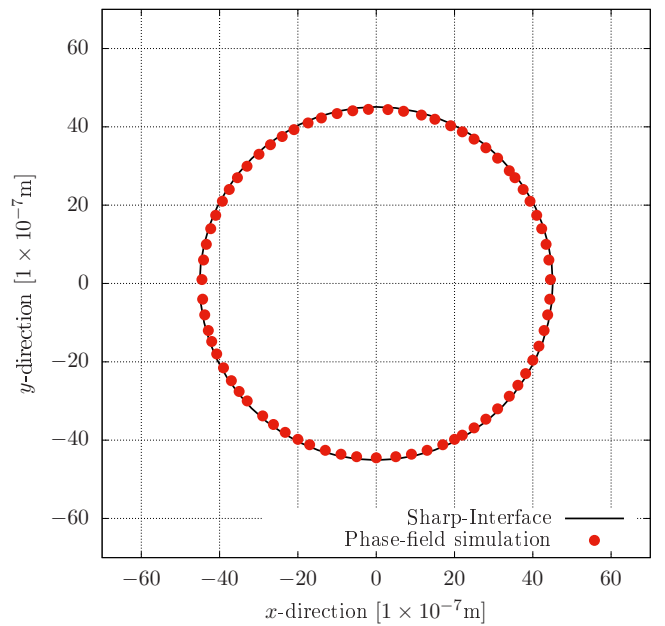

Figure 10. For a weak anisotropic solid-liquid interface, $\delta_{\alpha \beta}=0.005$, a direct comparison with the sharp-interface equilibrium shape shows excellent agreement, and the obtained effective anisotropy $\delta_{\alpha \beta}^{e}=0.0045$ shows a minute variation when compared with the imposed value.

\section{Conclusions}

In the present work, we have extensively discussed the unidirectional solidification of tip-splitting microstructures through the phase-field modeling approach. Here, the formation as well as the temporal evolution of morphological instabilities along a solidifying interface as a result of constitutional supercooling near the solid-liquid interface is simulated. At first, we have shown that the presence of grain boundaries in bicrystal alloys promotes ridge-shaped morphological instabilities near the grain boundary grooves. The convex instabilities across the solidification front consequently amplify and develop 
into a tip-splitting microstructure for energetically isotropic interfaces. With an increase in the ratio $R_{0}$, the role of the groove shape on the amplification of ridge-shaped instabilities near a grain boundary is demonstrated to be qualitatively in agreement with previous studies. Next, we have studied the tip-splitting phenomenon in a microstructural pattern, whereby the sharp-interface analytical criterion operates as the focal point for analyzing and predicting the tip-splitting position. The tip-splitting phenomenon builds upon the previous experimental and theoretical studies, and it successfully provides the regions for tip splitting for the first time.

The analytically obtained tip-splitting position is dynamically validated via performing two-dimensional phase-field simulations. Furthermore, the advancing interface tries to split in the same region as evaluated analytically. As a consequence of heating and cooling, the interface starts to evolve via forking between the positions, i.e., where the $\nabla_{S}^{2} \kappa$ passes through zero. The solidifying interface amplifies into the melt as a crest where $\nabla_{s}^{2} \kappa>0$, and it concurrently evolves as a trough where $\nabla_{s}^{2} \kappa<0$. Moreover, since the present mechanism is curvature dependent, the absence of tip-splitting events is also illustrated for a sphere, where the interfacial curvature varies linearly with its arc length. The effect of lattice anisotropy on the crystal-melt interfaces is also investigated, where we have successfully compared the equilibrium shapes from the phase-field simulations with its corresponding shape in the sharp-interface theory. In the end, through various numerical simulations and validation studies, we conclude by suggesting that through the present analytical criterion, a tip-splitting phenomenon is deterministic and predictable vis-á-vis the local interfacial curvature of the crystal, which should vary non-linearly with its arc length.

Supplementary Materials: The following supporting information can be downloaded at: https: / / www.mdpi.com/article/10.3390/met12030376/s1.

Author Contributions: Conceptualization, V.P.L., F.W.; Methodology, V.P.L., F.W.; Validation, V.P.L., F.W.; Investigation, V.P.L., F.W.; Software, V.P.L., F.W., M.S.; Visualization, V.P.L.; Writing-original draft preparation, V.P.L.; Writing-review and editing, V.P.L., F.W., M.S., B.N.; Supervision, B.N. All authors have read and agreed to the published version of the manuscript.

Funding: This research was funded by the cooperative graduate school "Gefügestrukturanalyse und Prozessbewertung" funded by the ministry of the state of Baden-Württemberg (MWK), Germany.

Institutional Review Board Statement: Not applicable.

Informed Consent Statement: Not applicable.

Data Availability Statement: The data presented in this study are available on request from the authors.

Acknowledgments: We would like to acknowledge financial support through the Gottfried-WilhelmLeibniz prize NE 822/31-1 of the research work by the German Research Foundation (DFG): Contributions to the classification of the tip-splitting events are provided by the programmes: Virtual Materials Design (VirtMat), project no. 9 (P3 MSE, 43.31.02), and Renewable 383 Energies (MTET 38.04.04) funded by the Helmholtz association. The authors also acknowledge the computing time from the high-performance clusters provided by the state of Baden-Württemberg through bwHPC.

Conflicts of Interest: The authors declare no conflict of interest.

\section{References}

1. Ihle, T.; Müller-Krumbhaar, H. Fractal and compact growth morphologies in phase transitions with diffusion transport. Phys. Rev. E 1994, 49, 2972. [CrossRef]

2. Ben-Jacob, E.; Deutscher, G.; Garik, P.; Goldenfeld, N.D.; Lareah, Y. Formation of a dense branching morphology in interfacial growth. Phys. Rev. Lett 1986, 57, 1903. [CrossRef]

3. Ben-Jacob, E.; Cohen, I.; Levine, H. Cooperative self-organization of microorganisms. Adv. Phys. 2000, 49, 395-554. [CrossRef]

4. Saffman, P.G.; Taylor, G. The penetration of a fluid into a porous medium or Hele-Shaw cell containing a more viscous liquid. Proceedings of the Royal Society of London A: Mathematical, Physical and Engineering Sciences. R. Soc. 1958, 245, $312-329$. 
5. Assadi, H.; Oghabi, M.; Herlach, D.M. Influence of ordering kinetics on dendritic growth morphology. Acta Mater. 2009, 57, 1639-1647. [CrossRef]

6. Glicksman, M.E. Principles of Solidification: An Introduction to Modern Casting and Crystal Growth Concepts; Springer Science \& Business Media: Berlin/Heidelberg, Germany, 2010.

7. Schaefer, R.; Glicksman, M. Initiation of dendrites by crystal imperfections. Metall. Trans. 1970, 1, 1973-1978. [CrossRef]

8. Mullins, W.W.; Sekerka, R. Stability of a planar interface during solidification of a dilute binary alloy. J. Appl. Phys. 1964, 35, 444-451. [CrossRef]

9. Mullins, W.W.; Sekerka, R.F. Morphological stability of a particle growing by diffusion or heat flow. J. Appl. Phys. 1963, 34, 323-329. [CrossRef]

10. Noël, N.; Jamgotchian, H.; Billia, B. In situ and real-time observation of the formation and dynamics of a cellular interface in a succinonitrile- $0.5 \mathrm{wt} \%$ acetone alloy directionally solidified in a cylinder. J. Cryst. Growth 1997, 181, 117-132. [CrossRef]

11. Morris, L.; Winegard, W. The development of cells during the solidification of a dilute Pb-Sb alloy. J. Cryst. Growth 1969, 5, 361-375. [CrossRef]

12. Utter, B.; Ragnarsson, R.; Bodenschatz, E. Alternating tip splitting in directional solidification. Phys. Rev. Lett. 2001, 86, 4604-4607. [CrossRef]

13. Nittmann, J.; Stanley, H.E. Tip splitting without interfacial tension and dendritic growth patterns. Nonlinear Phys. Beginners Fractals Chaos Solitons Pattern Form. Cell. Autom. Complex Syst. 1998, 321, 166.

14. Akamatsu, S.; Faivre, G.; Ihle, T. Symmetry-broken double fingers and seaweed patterns in thin-film directional solidification of a nonfaceted cubic crystal. Phys. Rev. E 1995, 51, 4751. [CrossRef]

15. Šebestíková, L.; D’Hernoncourt, J.; Hauser, M.; Müller, S.; De Wit, A. Flow-field development during finger splitting at an exothermic chemical reaction front. Phys. Rev. E 2007, 75, 026309. [CrossRef]

16. Glicksman, M.E. Mechanism of dendritic branching. Metall. Mater. Trans. A 2012, 43, 391-404. [CrossRef]

17. Martin, O.; Goldenfeld, N. Origin of sidebranching in dendritic growth. Phys. Rev. A 1987, 35, 1382. [CrossRef]

18. Devauchelle, O.; Szymczak, P.; Pecelerowicz, M.; Cohen, Y.; Seybold, H.J.; Rothman, D.H. Laplacian networks: Growth, local symmetry, and shape optimization. Phys. Rev. E 2017, 95, 033113. [CrossRef]

19. Suekane, T.; Ono, J.; Hyodo, A.; Nagatsu, Y. Three-dimensional viscous fingering of miscible fluids in porous media. Phys. Rev. Fluids 2017, 2, 103902. [CrossRef]

20. Mullis, A.M. Spontaneous deterministic side-branching behavior in phase-field simulations of equiaxed dendritic growth. $J$. Appl. Phys. 2015, 117, 114305. [CrossRef]

21. Shang, S.; Guo, Z.; Han, Z. On the kinetics of dendritic sidebranching: A three dimensional phase field study. J. Appl. Phys. 2016, 119, 164305, [CrossRef]

22. Wang, Z.; Wang, J.; Yang, G. Phase-field investigation of effects of surface-tension anisotropy on deterministic sidebranching in solutal dendritic growth. Phys. Rev. E 2008, 78, 042601. [CrossRef] [PubMed]

23. Jia-Xue, Y.; Zhi-Jun, W.; Jun-Jie, L.; Jin-Cheng, W. Tip-splitting instability in directional solidification based on bias field method. Chin. Phys. B 2015, 24, 078107.

24. Glicksman, M.E. Capillary-mediated interface perturbations: Deterministic pattern formation. J. Cryst. Growth 2016, 450, 119-139. [CrossRef]

25. Nestler, B.; Garcke, H.; Stinner, B. Multicomponent alloy solidification: phase-field modeling and simulations. Phys. Rev. E 2005, 71, 041609. [CrossRef]

26. Choudhury, A.; Nestler, B. Grand-potential formulation for multicomponent phase transformations combined with thin-interface asymptotics of the double-obstacle potential. Phys. Rev. E 2012, 85, 021602. [CrossRef]

27. Laxmipathy, V.P.; Wang, F.; Selzer, M.; Nestler, B. Phase-field simulations of grain boundary grooving under diffusive-convective conditions. Acta Mater. 2021, 204, 116497. [CrossRef]

28. Laxmipathy, V.P.; Wang, F.; Selzer, M.; Nestler, B. A two-dimensional phase-field study on dendritic growth competition under convective conditions. Comput. Mater. Sci. 2021, 186, 109964. [CrossRef]

29. Provatas, N.; Wang, Q.; Haataja, M.; Grant, M. Seaweed to dendrite transition in directional solidification. Phys. Rev. Lett. 2003, 91, 155502. [CrossRef]

30. Utter, B.; Bodenschatz, E. Dynamics of low anisotropy morphologies in directional solidification. Phys. Rev. E 2002, 66, 051604, [CrossRef]

31. Laxmipathy, V.P.; Wang, F.; Selzer, M.; Nestler, B.; Ankit, K. Influence of melt convection on the morphological evolution of seaweed structures: Insights from phase-field simulations. Comput. Mater. Sci. 2019, 170, 109196. [CrossRef]

32. Coriell, S.; Sekerka, R. Morphological stability near a grain boundary groove in a solid-liquid interface during solidification of a binary alloy. J. Cryst. Growth 1973, 19, 285-293. [CrossRef]

33. Dantzig, J.; Di Napoli, P.; Friedli, J.; Rappaz, M. Dendritic growth morphologies in Al-Zn alloys-Part II: Phase-field computations. Metall. Mater. Trans. A 2013, 44, 5532-5543. [CrossRef]

34. Kessler, D.A.; Koplik, J.; Levine, H. Geometrical models of interface evolution. II. Numerical simulation. Phys. Rev. A 1984, 30, 3161. [CrossRef]

35. Brower, R.C.; Kessler, D.A.; Koplik, J.; Levine, H. Geometrical models of interface evolution. Phys. Rev. A 1984, $29,1335-1342$. [CrossRef] 
36. Voorhees, P.; Coriell, S.; McFadden, G.; Sekerka, R. The effect of anisotropic crystal-melt surface tension on grain boundary groove morphology. J. Cryst. Growth 1984, 67, 425-440. [CrossRef]

37. Cahn, J.; Hoffman, D. A vector thermodynamics for anisotropic surfaces-II. Curved and faceted surfaces. Acta Metall. 1974, 22, 1205-1214. [CrossRef] 